\title{
Human-Robot Collaboration Using Industrial Robots
}

\author{
Dario Antonelli ${ }^{*}$ and Giulia Bruno \\ DIGEP - Politecnico di Torino, Torino - Italy \\ *Corresponding author
}

\begin{abstract}
Human-Robot Collaboration aims at exploiting the different but complementary skills of both the human worker and the programmable machine. It requires a high degree of interaction between the two actors and it is accomplished by special devoted robots. The study shows it is possible to redesign existing industrial robotic cells for executing a number of collaborative actions, respecting safety requirements.
\end{abstract}

Keywords-human-robot collaboration; learning by demonstration; automatic assembly

\section{INTRODUCTION}

There is an increasing interest both from academic people and from robot manufacturers in the design of robots that are able to work side by side with human operators for the execution of complex industrial tasks. In a survey on innovative flexible approaches to automotive assembly technologies [1], considerable importance is given to cooperation among humans and robots. During complex assembly tasks, some "components require both precise handling and secondary assembly operations, such as inserting fasteners and connecting wire harnesses. Some of these tasks require the precision and speed of automation, while others benefit from the dexterity and intelligence of human operators." Human-Robot Collaboration (HRC) brings benefits to industrial applications in terms of speed, efficiency, better quality of the production and better quality of the workplace (ergonomic) [2].

As far as now, robotic automation was seldom applied in small batch productions because of the variety of products and of variable production schedules [3]. The workplace organization leans towards flexibility where manual systems are advantaged. Now, several robot manufacturers have introduced special robot architectures, named collaborative robots, allowing the human workers to execute their assembly and welding tasks in the same workplace as the robots. Examples are KUKA LBR [4] and ABB YuMi [5]. Unfortunately, these robots have limitations in terms of payload, velocity, strength that prevent their widespread use. Some of these limits are related to the necessity of respecting the Technical Specifications ISO 15066 in order to assure human worker safety.

Present study investigates the subset of HRC that exploits standard industrial robots and, with the help of new sensors and state of the art machine learning, allows for a safe interaction with the robot, paying a reasonable fee in reduced flexibility.

The field of study is the industrial manual and automatic assembly by welding. Small factories usually already have robotic welding cells next to their manual workstations. Small production volumes or tight delivery deadlines are accomplished with the manual assembly. The robots should be used for large productions but are often underutilized.

In order to demonstrate the feasibility of employing standard industrial robots in flexible assembly cells, a flexible robotic cell was installed in a laboratory of the Politecnico di Torino University. The cell is reconfigured differently for every new assembly process under study.

The reconfiguration follows a number of steps. First of all, to introduce HRC in a standard industrial robotic cell, operation procedures that can be mapped both on manual workstations and on automatic ones are introduced. The concepts behind the assembly process are represented by an ontology. Another ontology describes the relationships among the components of a robotic cell. The matching of the two ontologies, instantiated for a specific industrial process allows to determine the redesign of the robotic cell. Then the sensors to be used for the particular task are installed in the cell and connected to the robot. Examples of such sensors are: Artificial Vision Cameras, Motion Capture Infrared Cameras, Laser Scanners, Force and Torque sensors. The sensors are employed to answer to a number of different requirements and to assure a fair level of performance in terms of accuracy, safety, consistency, dependability and compliance [6].

\section{ONTOLOGIES FOR HRC}

The description of the organization of a HRC system cannot leave out of consideration an architecture framework which includes all the working activities. Though it should be possible to find links to similar contexts, this reference model is not defined yet.

Ontologies plays a fundamental role in knowledge management because they formally specifies the key concepts, properties, relationships, and axioms of a given domain [7]. Furthermore, ontologies are a great tool for diminishing the ambiguity in knowledge transfer among groups of different agents: humans, robots and machines. All of them share the same conceptualization. Two ontologies are necessary in this study. The first one, named CCORA, is an extension of a standard ontology for robotics and automation (CORA) by adding concepts related to collaborative cells. The second one, named MPRO, is a manufacturing process ontology, which contains concepts related to manufacturing processes and related machines, tools and parameters. The two ontologies are detailed in the following subsections. 


\section{A. Collaborative CORA (CCORA)}

The Ontologies for Robotics and Automation Working Group (ORA WG) define a core ontology for robotics and automation (CORA), which specifies the general concepts in this context [8]. CORA extends SUMO, the Suggested Upper Merged Ontology, which is an open source upper ontology, widely used in several domains. CORA focuses on defining a robot, along with the specification of other related entities. Between CORA and SUMO, there is the CORAX ontology, which represents concepts and relations commonly found subdomains but that are too general to be included in CORA [9].

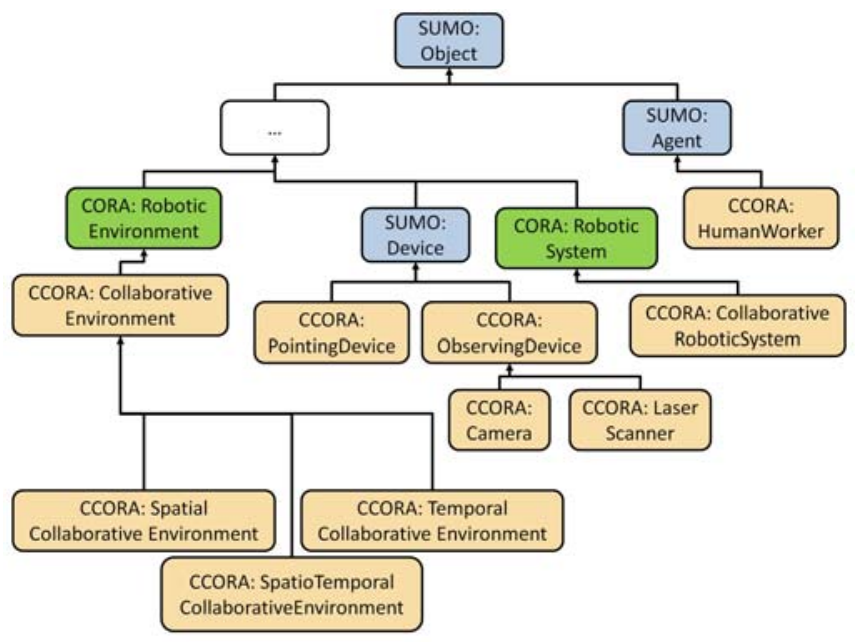

FIGURE I. CCORA CONCEPTS AND THEIR RELATIONSHIP WITH CORA AND SUMO

We further extended the CORA ontology in the Collaborative CORA (CCORA) by inserting the concepts related to human-robot collaboration, as shown in Figure 1. We define a collaborative robotic system an entity formed by robots, human workers, and a series of devices. Particularly, two kinds of devices are need: observing devices and pointing devices. A collaborative robotic environment, i.e., a collaborative cell, is an environment equipped with a collaborative robotic system. Three types of collaborative environments exist, the spatial collaboration environment, the temporal collaboration environment, and the spatiotemporal coloration environment. Depending on the kind of collaborative environement, different devices are needed. For example, in the case of a spatial collaboration, where humans and robots work in the same space but in different times, observing devices to reveal the human presence are needed, such as laser scanner, to grant that the human worker is not in the cell while the robot is working.

\section{B. Manufacturing Process (MPRO)}

The MPRO ontology cover the knowledge related to manufacturing operations. The main classes of the MPRO ontology Manufacturing process, Machine, Tool and Parameter [10]. A portion of the MPRO ontology related to the SUMO ontology is shown in Figure 2.

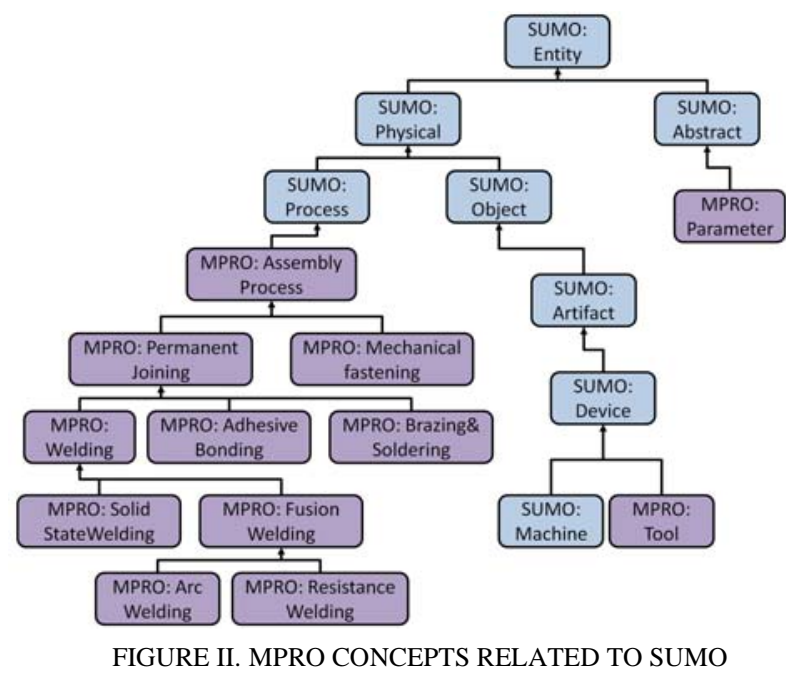

Manufacturing processes are divided into two main types: Processing operations and Assembly operations. Assembly operations are divided in Permanent joining processes and Mechanical fastening. Permanent joining processes are Welding, Brazing and soldering, and Adhesive bonding. Welding processes are further divided between Fusion welding and Solid state welding. To Fusion welding belong Arc Welding, Resistance Welding, Oxyfuel gas welding, and others.

The Machine class contains the information about the equipment that each process needs. The hierarchy of concepts of this class reflects the hierarchy of the manufacturing processes because the division of the machines is based on the process in which they are used. Tools are the elements that directly perform the work on the workpiece. Each tool has its own features such as size, material, shape, etc. The hierarchy of this class is defined with the same goal of the Machine class, enhancing the relationship between Manufacturing processes and Tools. Welding tools are used in different welding processes and include Welding molds, Solid state tools, and Fusion welding tools. Solid-state tools are Compression rolls, Rotation tools, and Ultrasonic transducers. Fusion welding tools are mostly related with Electrodes, which can be Consumables, if they are consumed during the process or Nonconsumables. Consumables electrodes are Coated electrodes, Electrogas welding electrode, Flux cored electrodes, Wire electrodes, and GMAW guns. Non-consumable electrodes are mostly Tungsten electrodes. The Parameter class represents the parameters of the manufacturing processes that have to be stored, such as the speed of the drill, the temperature, the water pressure, etc. In MPRO, five kinds of parameters are defined: Pressure, Sand mixture, Speed, Time and Temperature.

The two ontology are populated with different processes and provide the requirements for the design of every HRC work cell.

\section{THE SETUP OF THE HRC WORK CELL}

In the framework of the European Community funded project ECHORD FREE [11] a collaborative robot welding cell has been developed in the DIGEP laboratory at the Politecnico di Torino. Practically, the work cell was installed with all the 
exploitable smart sensors and robot controllers. Only the sensors that are required by the study of the process concept are activated in every specific case.

The choice of using standard industrial robots and the safety concerns leaded to one specific collaboration mechanism According to ISO/TS 15066, clause 5.10.4, the type of collaborative operation is speed and separation monitoring. It corresponds to the upper left box in Figure 3. The full collaboration (upper right box) would be obtained through power and force limiting by inherent design or control.

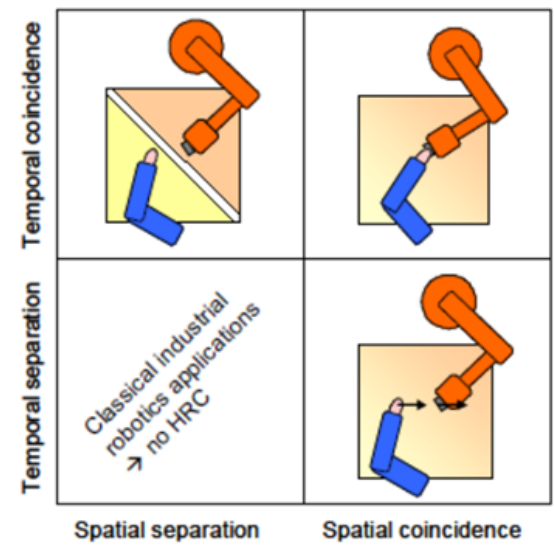

FIGURE III. TYPES OF COLLABORATIVE OPERATIONS (SOURCE ABB GROUP)

The conceptual scheme of the robotic cell is shown in Figure 6. In order to employ standard industrial robots, it was convenient to introduce a Communication Facilitator: a control loop operating at a level hierarchically superior than standard robot control, equipped with dedicated sensors, interfaced with the human operator and able to supervise the robot standard controller. The Facilitator receives visual images from optical cameras, measurement data from infrared sensors and presence in the working area of unexpected objects from two orthogonal laser scanners. Furthermore, it receives commands directly from the human co-worker. At symbolic level they are gestures, detected by a Leap Motion sensor. At trajectory level, it is the pose of a hand-held pointer tool. Facilitator performs the following tasks: Safety Manager, Programming by Demonstration, Mistake Detection.

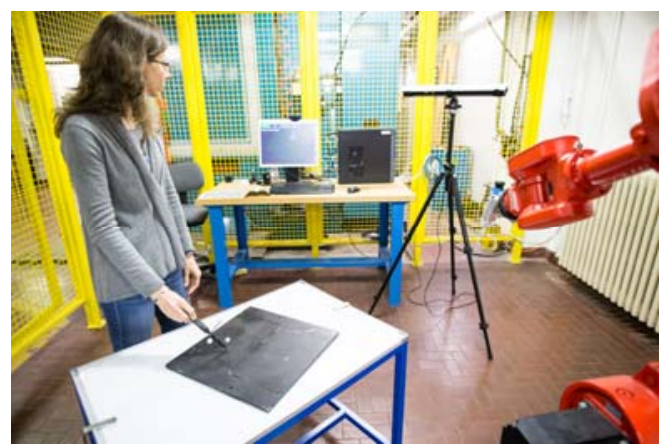

FIGURE IV. THE HRC CELL DURING THE ROBOT TRAINING

Figure 4 shows an example on a benchmark. The operator instructs the robot using a pointer equipped with IR reflective markers observed by the Optitrack motion capture. The robot, equipped with a custom felt-pen holder as end effector, will execute the program when the operator will go in the safe area.

\section{Proposed SOLUtion to HRC MAIn Issues}

\section{A. Safety}

The system is equipped with SICK laser scanners to identify the occurrence of events that modify the robot working safety conditions. Therefore, when events are identified, the Facilitator can actuate the control strategy able to keep the level of safety within the prefixed limits. Following the ISO/TS 15066, the system provides distance and speed supervision. The system forces a protective stop if the minimum separation distance or the speed limit is violated. The minimum separation distance is not fixed but is calculated by taking into account the braking time at current relative speed between the robot and the nearest human body section.

\section{B. Robot Programming}

Following [12] we can classify our problem as derivation of a policy by a mapping function approach that apply regression on a dataset built by the external observation of the demonstration through remote sensors.

In the path learning phase the operator moves a lightweight tool (a pointer) along a given trajectory. The action is recorded and reconstructed by the measuring sensor that produces a list of pointer poses. The list is used to produce a smooth and continuous approximation of the robot trajectory.

Derivation of the path from the measured points is not trivial [13]. Velocity data and their history is used to recognize stop-points. Multiple overlapping segments are merged and their points are properly reordered to obtain a single directed curve. Capability of the system to build a path from multiple overlapping segments is critical for industrial applications. There are many circumstances when indicating a path using just one continuous gesture is impossible or undesirable.

Optical acquisition produces noisy data points. The trajectory was approximated by a B-spline. Our aim is to produce welding trajectory with robot's accuracy, even if the teacher performs it with lower, human bounded, accuracy.

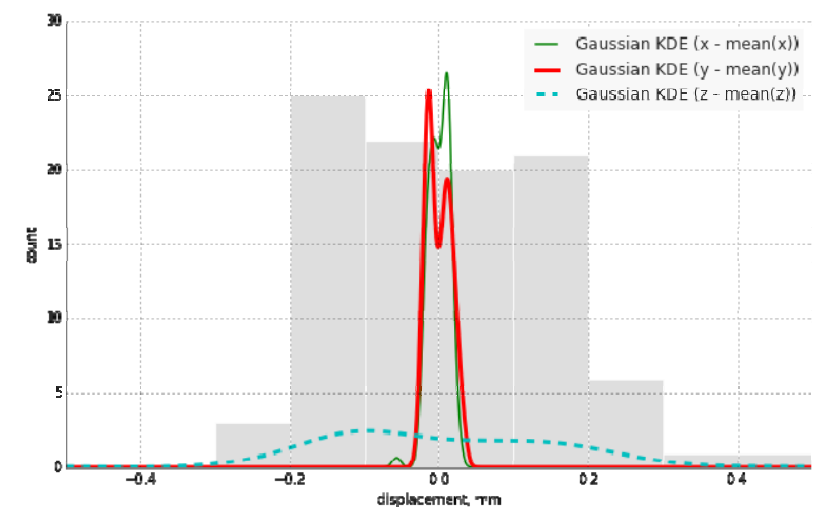

FIGURE V. KERNEL DENSITY ESTIMATES OF THE CALCULATED END-POINT LOCATION ACCURACY 


\section{Accuracy and Precision}

Repeatability of the end-point calculation for the pointer in the center of the frame was measured experimentally. The pointer was repeatedly put on an isostatic support, and its location was measured (Figure 6). The result indicates a good repeatability of the motion capture system. The repeatability in the $\mathrm{Z}$ direction (depth variable) is an order of magnitude worse as it is to be expected in stereo vision setups.

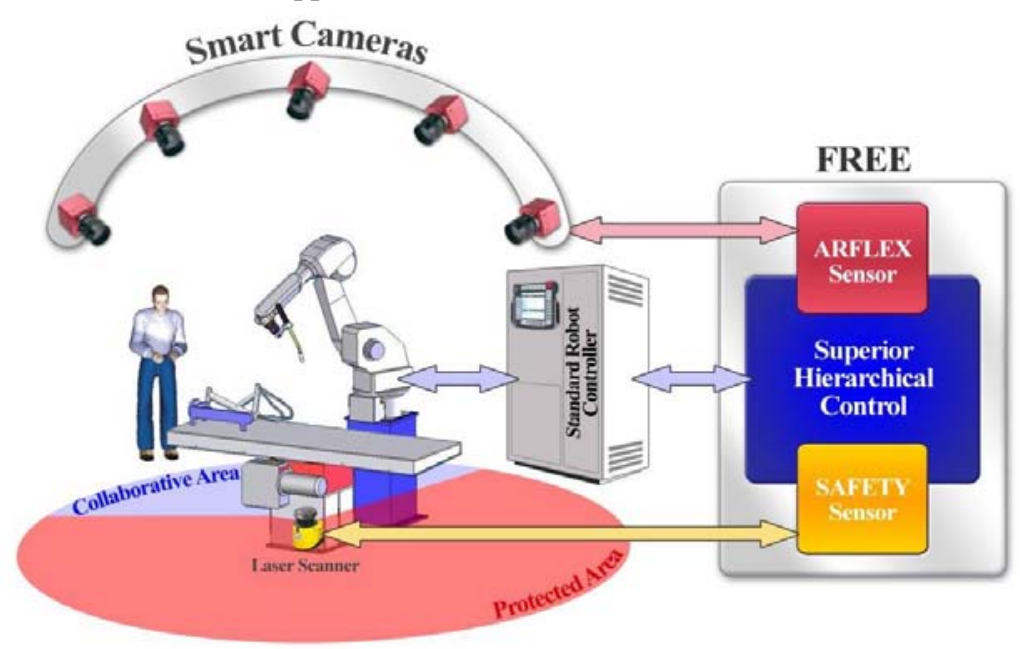

FIGURE VI. SCHEMATIC OVERVIEW OF THE HRC WORK CELL, SHOWING THE USE OF BOTH MEASURING AND SAFETY SENSORS

\section{CONCLUSIONS}

The study FREE aims at eliminating the barriers against industrial robot application in small batch production. Industrial applications require to assess if human demonstrations are interpreted and executed by the robot reliably and precisely. These aspects are rarely addressed in general Human-Robot Interaction research.

The entire system and individual components were qualified and benchmarked against the known human intent. While still improvable, the system can already be executed in a number of industrial scenarios.

\section{REFERENCES}

[1] Michalos, G., Makris, S., Papakostas, N., Mourtzis, D., \& Chryssolouris, G. (2010). Automotive assembly technologies review: challenges and outlook for a flexible and adaptive approach. CIRP Journal of Manufacturing Science and Technology, 2(2), 81-91.

[2] Helms, E., Schraft, R. D., \& Hagele, M. (2002). rob@ work: Robot assistant in industrial environments. In Robot and Human Interactive Communication, 2002. Proceedings. (pp. 399-404). IEEE.

[3] D. Antonelli, S. Astanin, G. Bruno (2016) Applicability of human-robot collaboration to small batch production, IFIP Advances in Information and Communication Technology, vol. 480, pp 24-32.

[4] Bischoff, R., Kurth, J., Schreiber, G., Koeppe, R., Albu-Schäffer, A., Beyer, A., ... \& Hirzinger, G. (2010, June). The KUKA-DLR Lightweight Robot arm-a new reference platform for robotics research and manufacturing. In Robotics (ISR), 2010 41st international symposium. VDE.

[5] Kirschner, D., Velik, R., Yahyanejad, S., Brandstötter, M., \& Hofbaur, M. (2016). YuMi, Come and Play with Me! A Collaborative Robot for Piecing Together a Tangram Puzzle. In International Conference on Interactive Collaborative Robotics (pp. 243-251). Springer.

[6] De Santis, A., Siciliano, B., De Luca, A., \& Bicchi, A. (2008). An atlas of physical human-robot interaction. Mechanism and Machine Theory, 43(3), 253-270.
[7] G. Bruno, D. Antonelli, R. Korf, N. Zimmermann "Exploitation of a Semantic Platform to Store and Reuse PLM Knowledge” IFIP Advances in Information and Communication Technology, 438, 2014, pp. 59-66.

[8] E. Prestes et al. "Towards a core ontology for robotics and automation" Robotics and Autonomous Systems, vol.61, 2013, pp.1193-1204.

[9] S. R. Fiorini et al. "Extensions to the core ontology for robotics and automation” Robotics and Computer-Integrated Manufacturing, vol. 33, 2015, pp.3-11.

[10] G. Bruno "Semantic organization of product lifecycle information through a modular ontology", International Journal of Circuits, Systems and Signal Processing, vol. 9, 2015, pp. 16-26.

[11] Antonelli D, Astanin S, Caporaletti G, Donati F. FREE: Flexible and Safe Interactive Human-Robot Environment for Small Batch Exacting Applications. Gearing Accel. Cross-Fertil. Acad. Ind. Robot. Res. Eur., Springer; 2014, p. 47-62.

[12] Argall, B. D., Chernova, S., Veloso, M., \& Browning, B. (2009). A survey of robot learning from demonstration. Robotics and Autonomous Systems, 57(5), 469-483.

[13] Fenghua, G., \& Caiming, Z. (2010, July). Curves reconstruction from ordered point cloud data. In Environmental Science and Information Application Technology (ESIAT), 2010 International Conference on (Vol. 2, pp. 84-87). IEEE. 